\title{
Rastreamento de hemoglobinas variantes e talassemias com associação de métodos de diagnóstico
}

\author{
Tracking of variant hemoglobins and thalassemias by association of diagnosis methodologies
}

Luciane M. S. Melo ${ }^{1}$

Fátima A. M. Siqueira ${ }^{1}$

Agnes C. F. Conte ${ }^{2}$

Claudia R. Bonini Domingos ${ }^{1}$
O diagnóstico neonatal de hemoglobinopatias permite a melhoria na qualidade de vida do doente com a implementação de medidas profiláticas, acompanhamento clínico e aconselhamento genético. Objetivou-se no presente estudo o diagnóstico das hemoglobinas variantes e talassemias em amostras de sangue de cordão umbilical de neonatos da região noroeste do estado de São Paulo por Cromatografia Líquida de Alta Performance (HPLC), associada a procedimentos eletroforéticos, bioquímicos e citológicos, visando adaptar a melhor metodologia de análise à freqüência dos defeitos de hemoglobina na população brasileira. Foram analisadas 3.048 amostras de janeiro de 2001 a dezembro de 2002, e 13,12\% apresentaram alterações de hemoglobinas, sendo $1,84 \%$ com presença de $\mathrm{Hb}$; ; $0,6 \% \mathrm{com} \mathrm{Hb} \mathrm{C}$; $0,65 \%$ com resultados sugestivos de beta talassemia e $9,48 \%$ sugestivos de alfa talassemia. Dentre as hemoglobinas anormais encontradas, 0,33\% das amostras apresentaram resultados discordantes nas metodologias aplicadas. A HPLC mostrou-se eficiente para a identificação de variantes de hemoglobinas e permitiu a análise de grande número de amostras em curto espaço de tempo e agilidade nas triagens. Entretanto, foi necessário associar outros métodos de análise para a caracterização das formas talassêmicas. Rev. bras. hematol. hemoter. 2008;30(1):12-17.

Palavras-chave: Triagem neonatal; hemoglobinopatias; talassemias; HPLC; diagnóstico laboratorial.

\section{Introdução}

As hemoglobinopatias são alterações genéticas com ampla distribuição mundial e, no Brasil, pelo alto grau de miscigenação da população, a incidência varia de acordo com a fixação regional dos principais grupos raciais colonizadores. ${ }^{1,2}$

Por sua diversidade genética e importância clínica, são consideradas problemas de saúde pública em muitos países inclusive o Brasil. Estão inseridas nos programas de triagem neonatal visando, através do diagnóstico precoce, a melhoria na qualidade de vida dos doentes com a implementação de medidas profiláticas e de condutas terapêuticas adequadas. Auxilia também nos procedimentos de orientação e acon- selhamento genético que, em um contexto educacional, permitem aos indivíduos ou famílias decidirem, de forma consciente e equilibrada, sobre a sua descendência. ${ }^{3,4}$ No Brasil, a inclusão das hemoglobinopatias nos programas de triagem neonatal foi efetivada em junho de 2001, com a portaria do Ministério da Saúde - no 822/GM. ${ }^{5}$

Em heterozigose, as hemoglobinas variantes geralmente não determinam manifestações clínicas significativas e as freqüências podem ser subestimadas. Dependendo da metodologia utilizada na caracterização, uma ou mais formas de hemoglobinas $(\mathrm{Hb})$ variantes podem aparecer com maior freqüência, e assim faz-se necessário estabelecer um padrão de análise para estas alterações genéticas. ${ }^{6,78}$

${ }^{1}$ Unesp - Ibilce - Laboratório de Hemoglobinas e Genética das Doenças Hematológicas (LHGDH).

${ }^{2}$ Famerp - Serviço de Genética - Faculdade de Medicina.

Trabalho desenvolvido no Laboratório de Hemoglobinas e Genética das Doenças Hematológicas - LHGDH - Depto. Biologia - Ibilce/Unesp.

Correspondência: Luciane Moreno Storti de Melo

Centro de Investigação de Microrganismos - CIM/Famerp - Bloco U6

Av. Brigadeiro Faria Lima, 5416 - São Manoel

15090-000 - São José do Rio Preto-SP - Brasil

Fone: (17) 3221-2390; Fax: (17) 3221-2390 - E-mail: stortilu@yahoo.com.br 
Objetivou-se, neste trabalho, realizar o diagnóstico de variantes de hemoglobinas e talassemias, em amostras de sangue de cordão umbilical, por metodologias clássicas de análise, como procedimentos eletroforéticos, bioquímicos e citológicos, comparados à Cromatografia Líquida de Alta Performance (HPLC) visando a adequação da melhor metodologia de análise às freqüências desses defeitos hereditários de Hb no Brasil.

\section{Casuística e Métodos}

A casuística constou de 3.048 amostras de sangue de cordão umbilical de recém-nascidos na região noroeste do estado de São Paulo, no período de janeiro de 2001 a dezembro de 2002, de ambos os gêneros e sem distinção étnica.

As amostras foram avaliadas inicialmente por métodos de triagem de hemoglobinopatias, tais como: Resistência globular osmótica em solução de $\mathrm{NaCl}$ a $0,36 \%,{ }^{9}$ um teste seletivo para rastreamento de talassemias do tipo beta; Eletroforese em acetato de celulose, $\mathrm{pH} 8,6,{ }^{10}$ que permite a separação de todas as hemoglobinas normais e grande parte das anormais; Análise da morfologia eritrocitária, ${ }^{3}$ para identificar alterações como hipocromia, microcitose, células em alvo e falcizadas; Cromatografia líquida de alta performance, HPLC, ${ }^{11}$ realizada com o Sistema automatizado Variant (BioRad), utilizando os reagentes e o protocolo do fabricante para esta faixa etária. O kit de diagnóstico do sistema foi especialmente desenhado para identificação de variantes de hemoglobinas na triagem neonatal a partir de sangue em papel filtro e foram realizadas as seguintes adequações para análise em sangue total: 5,0 $\mu \mathrm{L}$ de sangue de cordão diluídos em 1,0 mL de água destilada, homogeneizado por inversão e então utilizado na análise. Esta diluição apresentou a mesma concentração de $\mathrm{Hb}$ totais preconizada pelo fabricante.

Os resultados indicativos da presença de formas talassêmicas ou variantes de hemoglobinas foram submetidos aos seguintes testes complementares: Pesquisa de corpos de $\mathrm{Hb}$ H e Heinz, ${ }^{12}$ utilizada para a confirmação citológica destes agregados, após coloração vital, na suspeita de alfa talassemia e hemoglobinas instáveis; Eletroforese em ágar-fosfato, $\mathrm{pH}$ $6,2,{ }^{13}$ que permite separar as hemoglobinas $\mathrm{S}$ e $\mathrm{C}$ de outras variantes com padrão de migração semelhante em pH alcalino; e Eletroforese em pH neutro, ${ }^{14}$ para identificação das hemoglobinas Bart's e $\mathrm{H}$, indicativas de alfa talassemia nesta faixa etária. A análise estatística foi realizada, utilizando-se teste t de Student para comparação dos valores percentuais médios.

As amostras foram colhidas após consentimento informado, seguindo a orientação da Resolução 196/96. O projeto teve aprovação no comitê de ética em pesquisa da Unesp e Conep, registro 3919, processo 25000.034091/2002-88.

\section{Resultados}

Do total de 3.048 amostras de sangue de cordão umbilical avaliadas, 86,55\% apresentaram perfil de hemoglobinas normal para a idade com valores percentuais médios para $\mathrm{Hb}$ F de 73,01\% (DP 3,87) e valores mínimos e máximos 55,9\% e $84,1 \%$ respectivamente. Para $\mathrm{Hb} \mathrm{A}$, a média foi de 12,86\% (DP 4,14) valor mínimo de 2,4\% e máximo de 20,95\%, obtidos por HPLC. Foram encontradas 13,12\% das amostras com alterações de hemoglobinas que incluíram formas talassêmicas e variantes. Foram observados resultados discordantes nas metodologias de análise em $0,33 \%$ das amostras que tiveram resultados inconclusivos na triagem. Os diferentes fenótipos encontrados estão relacionados na tabela 1, e a figura 1 ilustra, para melhor visualização da distribuição, a representação gráfica desses fenótipos de hemoglobinas anormais, incluindo variantes e talassemias, sendo que o fenótipo de alfa talassemia heterozigota foi o mais freqüente com 9,48\%.

Para a triagem e identificação de hemoglobinas variantes realizaram-se as eletroforeses em $\mathrm{pH}$ alcalino e ácido, onde as diferentes frações de hemoglobinas puderam ser separadas por suas características físico-químicas, associadas à HPLC, que possui alta sensibilidade para a identificação de frações presentes em pequenas quantidades

Tabela 1. Fenótipos de hemoglobinas observados em sangue de cordão umbilical de amostras da região noroeste do estado de São Paulo

\begin{tabular}{|c|c|c|c|c|c|c|c|c|c|}
\hline \multicolumn{10}{|c|}{ Perfil de Hemoglobinas } \\
\hline Período & $\begin{array}{c}\text { Total } \\
\text { analisado } \\
\end{array}$ & Normal & $\begin{array}{r}\text { Talassemia } \\
\text { heterozigota }\end{array}$ & $\begin{array}{r}\text { Talassemia } \\
\text { heterozigota }\end{array}$ & FAS & FAC & Raras & Interações & $\begin{array}{c}\text { Total de } \\
\text { anormais }\end{array}$ \\
\hline \multirow[t]{3}{*}{2001} & 1288 & 1121 & 127 & 06 & 18 & 05 & 03 - rápida & $02-\alpha / \beta \operatorname{tal}(0,15 \%)$ & 163 \\
\hline & & $87,00 \%$ & $9,86 \%$ & $0,47 \%$ & $1,40 \%$ & $0,38 \%$ & $0,23 \%$ & 01 - Hb S/ $\alpha$ tal $(0,08 \%)$ & $12,65 \%$ \\
\hline & & & & & & & & $01-\mathrm{Hb} \mathrm{S} / \beta$ tal $(0,08 \%)$ & \\
\hline \multirow[t]{4}{*}{2002} & 1760 & 1517 & 162 & 14 & 38 & 13 & 01- rápida & $03-\alpha / \beta$ tal $(0,17 \%)$ & 237 \\
\hline & & $86,20 \%$ & $9,20 \%$ & $0,80 \%$ & $2,15 \%$ & $0,73 \%$ & $0,06 \%$ & $04-\mathrm{Hb} \mathrm{S} / \alpha$ tal $(0,23 \%)$ & $13,46 \%$ \\
\hline & & & & & & & & $01-\mathrm{Hb} C / \beta$ tal $(0,06 \%)$ & \\
\hline & & & & & & & & $01-\mathrm{Hb} S / \alpha / \beta$ tal $(0,06 \%)$ & \\
\hline \multirow[t]{2}{*}{ Total } & 3048 & 2638 & 289 & 20 & 56 & 18 & 04 & 13 & 400 \\
\hline & & $86,55 \%$ & $9,48 \%$ & $0,65 \%$ & $1,84 \%$ & $0,60 \%$ & $0,13 \%$ & $0,42 \%$ & $13,12 \%$ \\
\hline
\end{tabular}




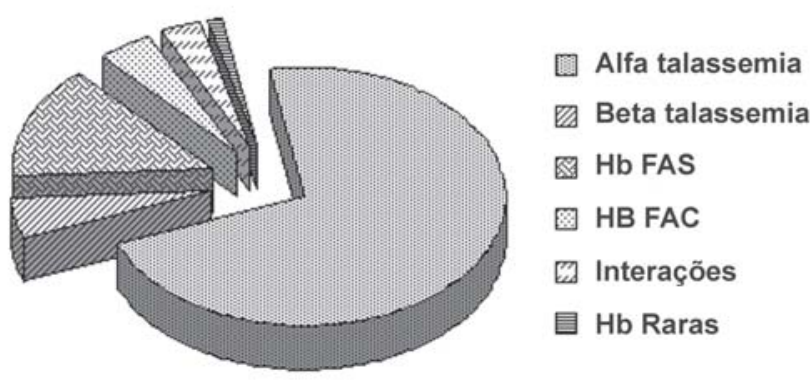

Figura 1. Representação gráfica dos fenótipos de hemoglobinas variantes e talassemias heterozigóticas encontrados no período de janeiro de 2001 a dezembro de 2002. O perfil normal foi suprimido para melhor visualização das frações anormais.

nessa faixa etária, como as variantes de cadeia beta, Hb S e $\mathrm{Hb}$ C. A figura 2 ilustra, em A, um cromatograma obtido por HPLC de uma amostra com $\mathrm{Hb}$ S, e, em B, as frações de hemoglobinas visualizadas em eletroforese em $\mathrm{pH}$ alcalino da mesma amostra.

Para as formas talassêmicas, a associação dos procedimentos de triagem e testes complementares, que incluíram a eletroforese em diferentes $\mathrm{pH}$, análise citológica e quantificação de frações, foi decisiva no estabelecimento dos fenótipos. A HPLC não foi utilizada como critério decisivo para o diagnóstico das talassemias, pois o Kit utilizado foi desenhado visando a identificação das hemoglobinas variantes $\mathrm{Hb}$ S, Hb C, Hb D e Hb E.

Para as suspeitas de alfa talassemia utilizaram-se as eletroforeses em $\mathrm{pH}$ alcalino e $\mathrm{pH}$ neutro para a visualização

\section{A}

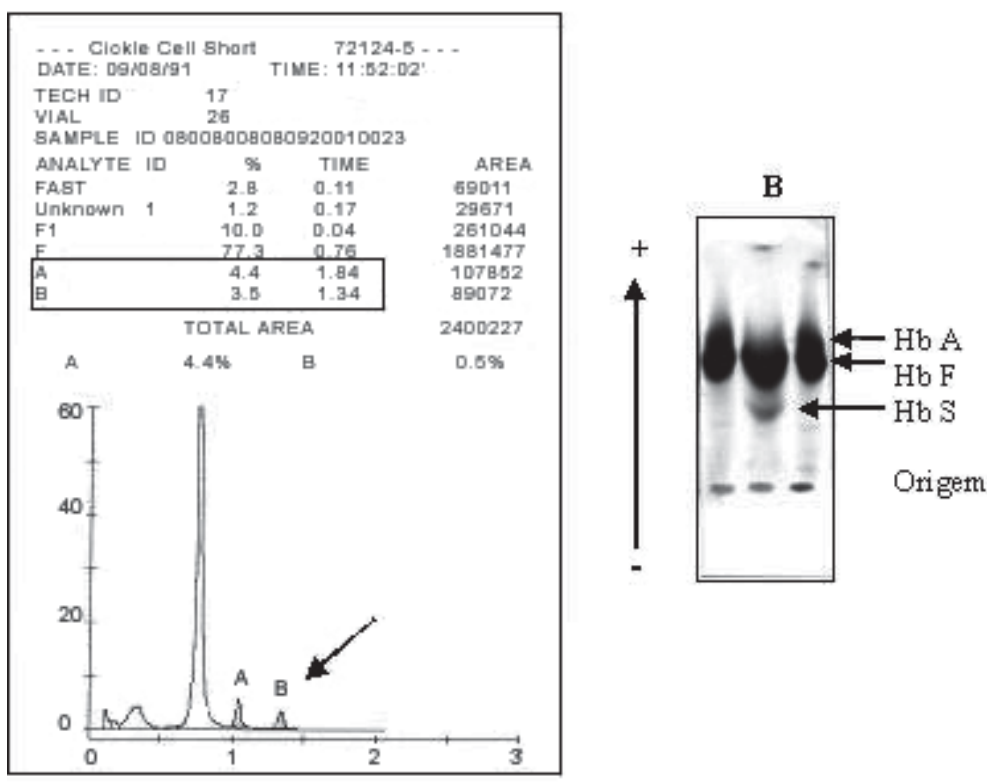

Figura 2. A - Cromatograma obtido pelo equipamento Variant (Bio-Rad) usando "Kit Sickle Cell Short Program" para HPLC. A seta indica a fração de Hb S. $B$ - Eletroforese em pH alcalino. As setas indicam as diferentes frações de hemoglobinas observadas
Tabela 2. Valores percentuais médios obtidos por HPLC para amostras normais e com alfa talassemia

\begin{tabular}{ccccc}
\hline & \multicolumn{2}{c}{ Pico "Fast" } & \multicolumn{2}{c}{ Pico "A" } \\
\hline X & DP & X & DP \\
\hline Normal & $2,64 \%$ & 1,64 & $12,86 \%$ & 4,14 \\
Alfa talassemia & $3,46 \%$ & 1,8 & $11,02 \%$ & 4,37 \\
\hline \multicolumn{2}{c}{ Valor $t$} & \multicolumn{2}{c}{6,96} & \multicolumn{2}{c}{6,41} \\
\hline$X=$ média, DP = desvio padrão
\end{tabular}

e confirmação das frações de hemoglobina Bart's e H, acrescidos da pesquisa intra-eritrocitária de corpos de $\mathrm{Hb} \mathrm{H}$. Os casos indicativos de alfa talassemia heterozigota, obtidos pela associação dessas metodologias, quando submetidos às análises por HPLC, mostraram o pico identificado como "Fast", estabelecido pelo programa, com valor percentual médio aumentado, sendo de 3,46\% (DP 1,8), e diminuição no valor percentual médio do pico de Hb A, 11,02\% (DP 4,37) em relação aos indivíduos com hemoglobinas normais para a idade, nos quais as médias percentuais foram de 2,64\% (DP 1,64) para o pico de "Fast" e 12,86\% (DP 4,14) para o pico de A.

Com a aplicação do teste $t$ de Student em nível de significância de 0,01 e valor crítico de $\pm 2,59$, a análise estatística rejeitou a hipótese de que os grupos seriam similares, mostrando diferença estatisticamente significativa entre as médias percentuais, sendo o valor $t=-6,96$ para o pico "Fast" e $t=6,41$ para o pico de A, como pode ser observado na tabela 2. O aumento observado no valor percentual médio do pico "Fast" das amostras indicativas de alfa talassemia heterozigota sugere que as $\mathrm{Hb}$ Bart's e $\mathrm{Hb} \mathrm{H}$ foram eluídas nos tempos de retenção (TR) que incluem frações de hemoglobina como a Hb F acetilada e produtos de degradação das amostras colhidas em papel.

Na figura 3 os valores percentuais e do TR dos picos "Fast" e A podem ser observados em cromatogramas com amostras de sangue normal e com alfa talassemia heterozigota e em $\mathrm{C}$ pode ser observada a presença da fração de Hb Bart's em eletroforese em $\mathrm{pH}$ neutro, um dos testes complementares utilizados para identificar esse tipo de hemoglobina.

Para a determinação dos casos de beta talassemia heterozigota, os critérios utilizados consistiram na associação dos resultados obtidos pela resistência globular osmótica aumentada na solução de $\mathrm{NaCl}$ a 0,36\%, análise da morfologia eritrocitária, na qual observaram-se alterações discretas na morfologia, e eletroforese em $\mathrm{pH}$ alcalino apresentando valores de $\mathrm{Hb}$ A diminuídos e de $\mathrm{Hb} \mathrm{F}$ aumentados. Apesar do kit utilizado na HPLC não ser específico para o diagnóstico das talassemias, observou-se diminuição no valor percentual médio do pico de $\mathrm{Hb}$ A (4,68 \%, DP 2,0) e aumento na média percentual do pico de Hb F (80,85\%, DP 2,07) em 

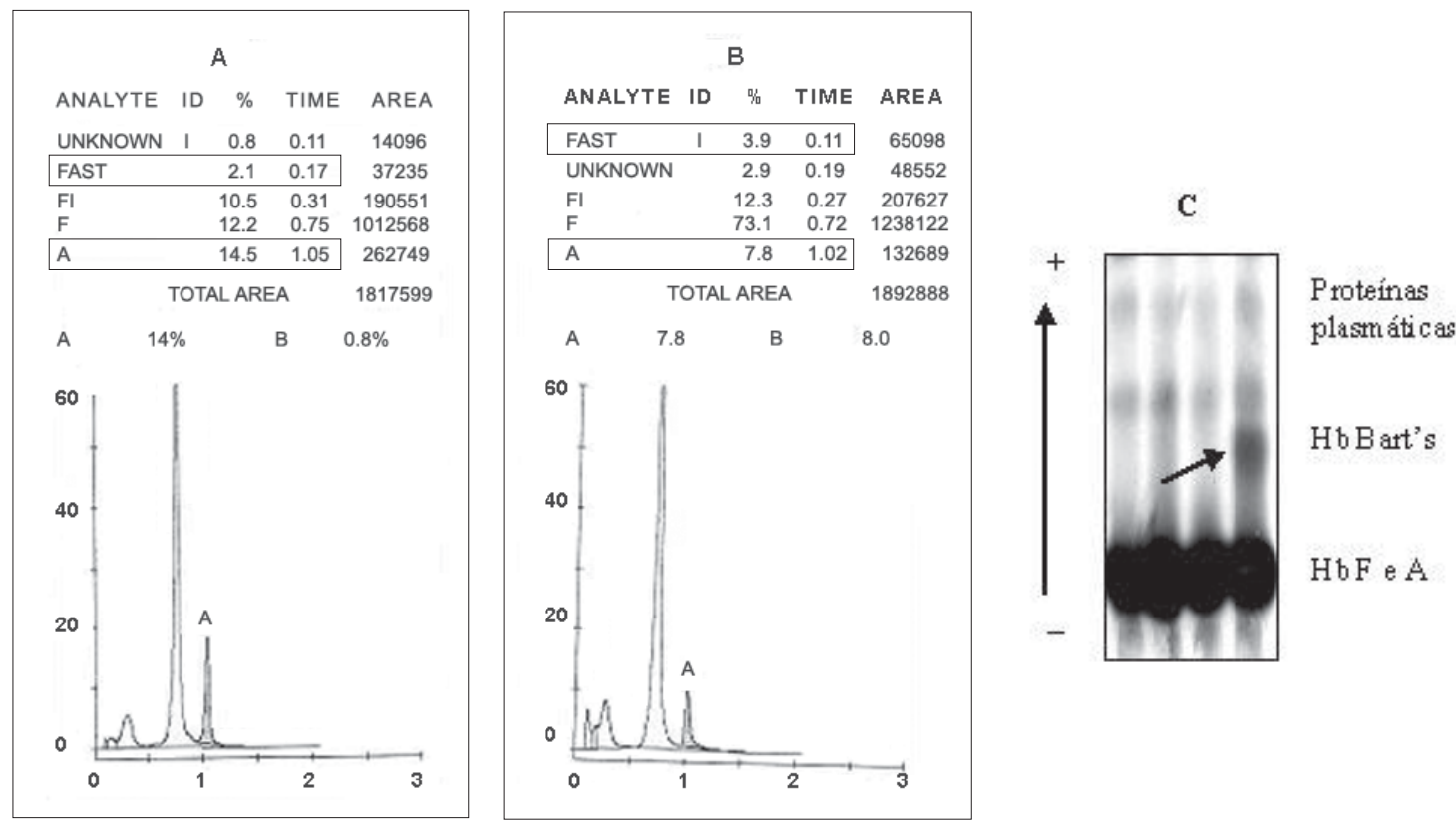

Figura 3. Cromatogramas obtidos pelo equipamento Variant (Bio-Rad) com "Kit Sickle Cell Short Program" de HPLC. A - amostra normal, B amostra com resultados indicativos de alfa talassemia heterozigota. Em destaque as janelas de "Fast" e "A". C - Eletroforese em acetato de celulose, $\mathrm{pH}$ neutro. A seta indica a presença da fração de hemoglobina Bart's

Tabela 3. Valores percentuais médios obtidos por HPLC para amostras normais e com beta talassemia

\begin{tabular}{ccccc}
\hline & \multicolumn{2}{c}{ Pico "Fast" } & \multicolumn{2}{c}{ Pico "A" } \\
\hline & X & DP & X & DP \\
\hline Normal & $73,01 \%$ & 3,87 & $12,86 \%$ & 4,14 \\
Beta talassemia & $80,85 \%$ & 2,07 & $4,68 \%$ & 2,0 \\
\hline Valor $t$ & \multicolumn{2}{c}{$-17,07$} & \multicolumn{2}{c}{18,36} \\
\hline
\end{tabular}

$\mathrm{X}=$ média; $\mathrm{DP}=$ desvio padrão

relação às amostras com hemoglobinas normais $(\mathrm{Hb} \mathrm{A}=$ $12,86 \%$ e Hb F = 73,01 \%). A aplicação de teste estatístico $t$ de Student com nível de significância de 0,01 e valor crítico de $\pm 2,82$ apresentou diferença estatisticamente significativa, com $t=17,07$ para o pico de $\mathrm{Hb} \mathrm{F} \mathrm{e} t=18,36$ para o pico de A, quando comparados com o grupo com hemoglobinas normais. A tabela 3 ilustra a comparação dos valores obtidos para essas amostras. Outros autores encontraram valores similares aos por nós observados, em grupos populacionais diversos e com coleta de amostra diversificada. ${ }^{15,16}$

Todos os resultados obtidos nos testes de triagem foram confirmados após os seis meses de vida, com estudos dos familiares.

\section{Discussão}

A partir de junho de 2001, a portaria do Ministério da Saúde $n^{\circ} 822$ tornou obrigatória a inclusão da anemia falciforme e outras hemoglobinopatias nos testes de triagem neonatal nos estados cadastrados nas fases II ou III do Pro- grama Nacional de Triagem Neonatal. Essa inclusão representou grande avanço na saúde pública do país, permitindo o diagnóstico precoce das alterações de hemoglobinas e assim contribuindo para a redução da morbi-mortalidade decorrente dessas alterações genéticas e promovendo a melhoria na qualidade de vida dos doentes. No entanto, deve-se ressaltar que as hemoglobinopatias são alterações com grande diversidade genética que incluem formas variantes e talassêmicas e, diante da freqüência das talassemias heterozigotas observadas na população brasileira, destaca-se a importância da padronização de técnicas para o diagnóstico dessas alterações, aumentando assim a abrangência do Programa Nacional. ${ }^{17}$ Os procedimentos eletroforéticos são bastante utilizados nas análises de hemoglobinas por serem capazes de separar todas as hemoglobinas normais e parte das anormais. No entanto, a maioria inclui técnicas não automatizadas e que dependem de inspeção visual para a determinação do fenótipo. As alterações de cadeia beta encontram-se expressas em pequenas quantidades no período neonatal e este fato representa uma dificuldade adicional para a visualização das frações nos procedimentos eletroforéticos usuais. $^{18}$

A HPLC é um método sensível, que possui limites de deteç̧ão aceitáveis, permitindo a sua aplicação na rotina de análise com controle de qualidade, sendo capaz de identificar e diferenciar várias hemoglobinas variantes. A HPLC apresenta características importantes para amplos programas de prevenção das hemoglobinopatias, como a rapidez nas análises, utilizando pequena quantidade de amostra, análise de um grande número de amostras ao mesmo tempo e o forneci- 
mento de resultados semiquantitativos, com custo por teste comparável a outros métodos existentes em laboratórios que trabalham com grandes volumes de amostras. ${ }^{19}$

A utilização das metodologias clássicas de diagnóstico de hemoglobinopatias associadas à sensibilidade da HPLC possibilitou, neste estudo, a identificação de aproximadamente 3,0\% de amostras com hemoglobinas variantes, incluindo as variantes mais freqüentes como as $\mathrm{Hb} \mathrm{S}$ e $\mathrm{Hb} \mathrm{C}$, a associação dessas às formas talassêmicas e ainda hemoglobinas de rara freqüência, refletindo a diversidade genética da população brasileira.

Para o diagnóstico das talassemias, fez-se necessária a utilização conjunta da HPLC e de metodologias clássicas de análise quantitativas e qualitativas, que foram decisivas na identificação das alterações características de formas talassêmicas. AHPLC não foi utilizada como critério diagnóstico decisivo para as talassemias, mas, apesar de não ser capaz de identificar e quantificar precisamente as Hb Bart's e $\mathrm{H}$, observou-se um aumento percentual médio de frações com baixo tempo de eluição, como o pico identificado como "Fast", naqueles cromatogramas de amostras com suspeita de alfa talassemia, sendo a variação nesses valores estatisticamente significativa. Essa observação auxiliou o direcionamento das análises, permitindo a pesquisa cuidadosa nos testes específicos para identificação de alfa talassemia heterozigótica.

Da mesma forma, a HPLC também não foi determinante no diagnóstico dos casos de beta talassemia heterozigótica, o qual foi baseado nos resultados conjuntos obtidos nas diferentes metodologias de análise. No entanto, ao comparar os valores de $\mathrm{Hb} \mathrm{A}$ e $\mathrm{Hb}$ F, obtidos por HPLC, nos portadores de beta talassemia heterozigota com os valores percentuais médios encontrados em indivíduos normais, observou-se diminuição na média percentual de $\mathrm{Hb}$ A e aumento no valor percentual médio da $\mathrm{Hb}$ F, sendo essa diferença estatisticamente significativa. Esses achados foram compatíveis com relatos de talassemia do tipo beta rastreados nessa fase, ${ }^{15,16,}$ ${ }^{20}$ sendo esta uma observação importante para auxiliar a suspeita diagnóstica e o direcionamento das análises complementares. Devido ao baixo número de amostras encontradas com esta hemoglobinopatia, não foi possível estabelecer um padrão de análise confiável somente por HPLC nesse kit específico. Para a correta caracterização das formas talassêmicas, todas as frações de hemoglobinas devem ser avaliadas em diferentes procedimentos laboratoriais e os casos suspeitos confirmados em reteste após seis meses de vida, sendo que a avaliação global pela HPLC fornece indícios para análises complementares.

A confirmação diagnóstica após seis meses de idade permitiu a realização do aconselhamento genético de caráter não diretivo, com atuação de equipe multiprofissional, auxiliando no acompanhamento de cada caso, fornecendo explicações sobre o que são anemias hereditárias, como são transmitidas e a importância do diagnóstico precoce para os familiares. Destacou-se o interesse crescente da comunidade em comparecer às sessões de aconselhamento e em obter informações, trazendo outros familiares para realizar o diagnóstico, possibilitando a divulgação de sua importância para a comunidade. Com os retestes e estudos familiares, as suspeitas iniciais da presença de hemoglobinopatias foram confirmadas corroborando os achados laboratoriais anteriores.

Através da análise de amostras de sangue de cordão umbilical por procedimentos cromatográficos, eletroforéticos e citológicos, sugeriu-se que os resultados em conjunto favoreceram o direcionamento laboratorial para o diagnóstico dos diferentes tipos de alterações das hemoglobinas incluindo as talassemias. Em programas de triagem neonatal, considerando-se a característica étnica da população brasileira, a aplicação de diferentes metodologias permitiu uma pesquisa completa de hemoglobinas, favorecendo a orientação genética e educacional adequada a cada caso.

\section{Abstract}

The neonatal diagnosis hemoglobinopathies improves the quality of life by prophylactic measures and genetic counseling. The diagnosis of variant hemoglobins and thalassemias was considered in the present study. Cord blood samples of newborn babies from the northwestern region of São Paulo state were analyzed by High Performance Liquid Chromatography (HPLC) associated with electrophoretic, biochemical and cytologic procedures aiming to adapt the best methodology to analyze the frequency of hemoglobin defects in the Brazilian population. Three thousand and forty-eight samples were analyzed from January 2001 to December 2002 with $13.12 \%$ presenting hemoglobin alterations; $1.84 \%$ had $\mathrm{Hb} \mathrm{S;} 0.6 \%$ had $\mathrm{Hb}$ C; $0.65 \%$ were suggestive of thalassemia beta and $9.48 \%$ were suggestive of thalassemia alpha. Among the abnormal hemoglobins, $0.33 \%$ of the samples presented different results in the methodologies used. HPLC was efficient to identify variant hemoglobins and enable the analysis of several samples in a short period of time with agility in screenin. However, an association of other methods was necessary for the characterization of the thalassemic forms. Rev. bras. hematol. hemoter. 2008;30(1):12-17.

Key words: Neonatal screening; hemoglobinopathies; thalassemias; HPLC; laboratorial diagnosis

\section{Agradecimentos}

À Bio-Oxford e Bio-Rad pelo apoio nas análises cromatográficas. À Pró-Reitoria de Extensão da Unesp e à Capes pelas bolsas de estudos fornecidas.

\section{Referências Bibliográficas}

1. Zago MA. Hemoglobinopatias: prevalência e variabilidade. Rev Paul Med 1986; 6:300-04.

2. Naoum PC. Hemoglobinopatias e talassemias. São Paulo: Sarvier; 1997.

3. Bonini-Domingos CR. Hemoglobinopatias no Brasil: variabilidade genética e metodologia laboratorial [Tese de Doutorado]. São José do Rio Preto: Instituto de Biociências Letras e Ciências Exatas, Universidade Estadual Paulista; 1993. 
4. Ramalho AS, Magna LA, Silva RBP. A Portaria MS no 822/01 e a triagem neonatal das hemoglobinopatias. Rev Bras Hematol Hemoter. 2002;4:244-50.

5. Brasil, Ministério da Saúde. Portaria nº 822, 6 de junho de 2001. Brasília, 2001.

6. Bonini-Domingos CR, Mendes-Siqueira FA. Diagnóstico laboratorial das hemoglobinopatias. Newslab. 2000;42:70-6.

7. Naoum PC. Prevalência e controle da Hb S. Rev Bras Hematol Hemoter. 2000;1.2:142-8.

8. Bonini-Domingos $\mathrm{CR}$ et al. Estudo de $\mathrm{Hb}$ anormais em doadores de sangue e recém-nascidos de São José do Rio Preto, SP. Newslab. 2000;41:92-8.

9. Silvestroni E, Bianco I. Screening for microcytemia in Italy: analysis of data collected in the past 30 years. Am J Hum Genet. 1975; 27: 198-212.

10. Marengo-Rowe AJ. Rapid electrophoresis and quantitation of haemoglobin on cellulose acetate. J Clin Path. 1965;18:790-2.

11. Bio Rad- Protocols for HPLC Variant Sickle cell short Program.

12. Papayannopoulos R, Stamatonyannopoulos G. Stains for inclusions bodies. In: Standartization of laboratory reagents and methodos for detection of haemoglobinopathies. Atlanta: Hew publications; 1974.

13. Vella F. Acid agar gel electrophoresis of human hemoglobin. Am J Clin Path. 1968;3:440-2.

14. Dacie JV, Lewis SM. Practical Haematology. $6^{\text {th }}$ ed. London: Churchill; 1985.

15. Fucharoen S, Winichagoon P, Wisedpanichkij R, Sae-Ngow B, Sriphanich R, Oncoung W et al. Prenatal and postnatal diagnoses of thalassemias and hemoglobinopathies by HPLC. Clin Chem. 1998;4:740-8.

16. van der Dijs FP, van den Berg GA, Schermer JG, Muskiet FD, Landman H, Muskiet FA. Screening cord blood for hemoglobinopathies and thalassemia by HPLC. Clin Chem. 1992;9:1864-9.

17. Mendes-Siqueira FA. Contribuição para o estudo das alterações moleculares e interferentes na expressão fenotípica das hemoglobinopatias a partir de um programa de diagnóstico neonatal [Tese de Doutorado]. São José do Rio Preto: Instituto de Biociências Letras e Ciências Exatas, Universidade Estadual Paulista; 2004.

18. Eastman JW, Wong R, Liao CL, Morales DR. Automated HPLC screening of newborns for sickle cell anemia and other hemoglobinopathies. Clin Chem. 1996;5:704-10.

19. Tan GB, Aw TC, Dunstan RA, Lee SH. Evaluation of high performance liquid chromatography for routine estimation of haemoglobins A2 and F. J Clin Pat. 1993;46:852-6.

20. van der Dijs FP, Volmer M, van Gijssel-Wiersma DG, Smit JW, van Veen R, Muskiet FA. Predictive value of cord blood hematological indices and hemoglobin Barts for the detection of heterozygous alpha-thalassemia-2 in an African-Caribbean population. Clin Chem. 1999;9:1495-500.

Avaliação: Editor e dois revisores externos

Conflito de interesse: sem conflito de interesses.

Recebido: 07/08/2006

Aceito após modificações: 19/09/2007 